$7^{\text {th }}$ Jagna International Workshop (2014)

International Journal of Modern Physics: Conference Series

Vol. 36 (2015) 1560015 (5 pages)

(C) The Authors

DOI: 10.1142/S2010194515600150

\title{
Lévy path integral approach to the fractional Schrödinger equation with delta-perturbed infinite square well
}

\author{
M. M. I. Nayga* and J. P. H. Esguerra \\ National Institute of Physics, University of the Philippines-Diliman, \\ Quezon City 1101, Philippines \\ *mnayga@nip.upd.edu.ph
}

Published 2 January 2015

\begin{abstract}
Using a path integral approach, we consider a fractional Schrödinger equation with deltaperturbed infinite square well. The Lévy path integral, which is generalized from the Feynman path intergal for the propagator, is expanded into a perturbation series. From this, the energy-dependent Green's function is obtained.
\end{abstract}

Keywords: Fractional quantum mechanics; path integral.

PACS Number: 03.65.Db

\section{Introduction}

Fractional quantum mechanics was first introduced by Laskin. It is described by the space-fractional Schrödinger equation (SFSE) containing the Riesz fractional operator. Following Feynman's path integral approach to quantum mechanics, Laskin generalized the path integral over Brownian motions to Lévy flights and obtained the space-fractional Schrödinger equation. ${ }^{1,2}$

Solutions to the space-fractional Schrödinger equation with linear potential, delta potential, infinite square well, and Coulumb potential, have already been obtained via piece-wise solution approach, momentum representation method, and, indirectly, the Lévy path integral approach. ${ }^{3-5}$ However, despite the numerous works on fractional quantum mechanics, perturbation has not yet been explored. In this paper, we consider the space-fractional Schrödinger equation with perturbative terms using the Lévy path integral approach. We follow Grosche's perturbation expansion scheme ${ }^{6,7}$ and obtained an energy-dependent Green's function for delta perturbations. As an example, we consider an infinite square well with delta-function perturbation.

This is an Open Access article published by World Scientific Publishing Company. It is distributed under the terms of the Creative Commons Attribution 3.0 (CC-BY) License. Further distribution of this work is permitted, provided the original work is properly cited. 


\section{Lévy path integral and fractional Schrödinger equation}

If a particle starts from a point $x_{i}$ at an initial time $t_{i}$ and goes to a final point $x_{f}$ at time $t_{f}$, its path $x(t)$ will have the property, $x\left(t_{i}\right)=x_{i}$ and $x\left(t_{f}\right)=x_{f}$. To get from the initial point to the final point, we define a propagator, $K_{L}\left(x_{f}, x_{i} ; t_{f}-t_{i}\right)$, which is the sum over all of the paths that go between points $\left(x_{i}, t_{i}\right)$ and $\left(x_{f}, t_{f}\right)$. If a particle moves in a potential, then the propagator is written as

$$
K_{L}\left(x_{f}, x_{i} ; t_{f}-t_{i}\right)=\int_{x\left(t_{i}\right)=x_{i}}^{x\left(t_{f}\right)=x_{f}} D x\left(t^{\prime}\right) \exp \left[-\frac{i}{\hbar} \int_{t_{i}}^{t_{f}} d t^{\prime} V\left(x\left(t^{\prime}\right)\right)\right],
$$

where $V\left(x\left(t^{\prime}\right)\right)$ is the potential energy as a functional of the Lévy particle path and the fractional path integral measure is defined $\mathrm{as}^{2}$

$$
\begin{aligned}
\int_{x\left(t_{i}\right)=x_{i}}^{x\left(t_{f}\right)=x_{f}} D x\left(t^{\prime}\right)= & \lim _{N \rightarrow \infty} \int d x_{1} \ldots d x_{N-1}\left(\frac{i D_{\alpha} \epsilon}{\hbar}\right)^{-N / \alpha} \\
& \times \prod_{j=1}^{N} L_{\alpha}\left[\left(\frac{\hbar}{i D_{\alpha} \epsilon}\right)^{1 / \alpha}\left|x_{j}-x_{j-1}\right|\right] \ldots,
\end{aligned}
$$

where $D_{\alpha}$ is the generalized 'fractional diffusion coefficient' (has physical dimension, $\left[D_{\alpha}\right]=\mathrm{erg}^{1-\alpha} \mathrm{cm}^{\alpha} \mathrm{s}^{\alpha}, D_{\alpha}=1 / 2 \mathrm{~m}$ for $\alpha=2$, m denotes the mass of the particle), $x_{0}=x_{i}, x_{N}=x_{f}, \epsilon=\left(t_{f}-t_{i}\right) / N$, and $L_{\alpha}(x)$ is the Lévy probability distribution function. For $\alpha=2$, equation (2) is transformed to the Feynman free particle propagator. $^{2}$

The propagator describes the evolution of the fractional quantum mechanical system in the following way,

$$
\psi_{f}\left(x_{f}, t_{f}\right)=\int_{-\infty}^{+\infty} d x_{i} K_{L}\left(x_{f}, x_{i} ; t_{f}-t_{i}\right) \psi_{i}\left(x_{i}, t_{i}\right)
$$

where $\psi_{i}\left(x_{i}, t_{i}\right)$ is the fractional wave function of the initial state and $\psi_{f}\left(x_{f}, t_{f}\right)$ is the fractional wave function of the final state. Laskin derived the one-dimensional fractional Schrödinger equation as follows

$$
i \hbar \frac{\partial \psi(x, t)}{\partial t}=\left[-D_{\alpha}(\hbar \nabla)^{\alpha}+V(x, t)\right] \psi(x, t),
$$

where $(\hbar \nabla)^{\alpha}$ is the Riesz fractional derivative operator,

$$
(\hbar \nabla)^{\alpha} \psi(x, t)=-\frac{1}{2 \pi \hbar} \int_{-\infty}^{+\infty} d p e^{i p x / \hbar}|p|^{\alpha} \int_{-\infty}^{+\infty} e^{-i p x / \hbar} \psi(x, t) d x .
$$

\section{Path integration via summation of perturbation expansions}

We follow Grosche's method for the time-ordered perturbation expansion. ${ }^{6,7} \mathrm{We}$ assume that we have a potential $W(x)=V(x)+\tilde{V}(x)$. The propagator corresponding to $V(x)$ is assumed to be known. We expand the propagator containing $\tilde{V}(x)$ in a perturbation expansion about $V(x)$ in the following way. The initial kernel corresponding to $V(x)$ propagates in $\Delta t$ time unperturbed, then interacts with $\tilde{V}(x)$, 
propagates again in another $\Delta t$ time unperturbed, and so on, up to the final state. We obtain the following expansion ${ }^{6,7}$

$$
\begin{aligned}
K_{L}\left(x_{f}, x_{i} ; t_{f}-t_{i}\right)= & \int_{x\left(t_{i}\right)=x_{i}}^{x\left(t_{f}\right)=x_{f}} D x\left(t^{\prime}\right) \exp \left[-\frac{i}{\hbar} \int_{t_{i}}^{t_{f}} d t^{\prime}\left(V\left(x\left(t^{\prime}\right)\right)+\tilde{V}\left(x\left(t^{\prime}\right)\right)\right)\right] \\
= & \int_{x\left(t_{i}\right)=x_{i}}^{x\left(t_{f}\right)=x_{f}} D x\left(t^{\prime}\right) \exp \left[-\frac{i}{\hbar} \int_{t_{i}}^{t_{f}} d t^{\prime} V\left(x\left(t^{\prime}\right)\right)\right] \\
& +\sum_{n=1}^{\infty}\left(\frac{-i}{\hbar}\right)^{n} \frac{1}{n !} \int_{x\left(t_{i}\right)=x_{i}}^{x\left(t_{f}\right)=x_{f}} D x\left(t^{\prime}\right) \exp \left[-\frac{i}{\hbar} \int_{t_{i}}^{t_{f}} d t^{\prime} V\left(x\left(t^{\prime}\right)\right)\right] \\
& \times\left[\int_{t_{i}}^{t_{f}} d t^{\prime} \tilde{V}\left(x\left(t^{\prime}\right)\right)\right]^{n}
\end{aligned}
$$

Introducing a time-ordering operator, the expansion becomes

$$
\begin{aligned}
K_{L}\left(x_{f}, x_{i} ; t_{f}-t_{i}\right)= & K_{L}^{(V)}\left(x_{f}, x_{i} ; t_{f}-t_{i}\right)+\sum_{n=1}^{\infty}\left(\frac{-i}{\hbar}\right)^{n}\left[\prod_{k=1}^{n} \int_{t_{i}}^{t_{k}} d t_{k} \int_{-\infty}^{+\infty} d x_{k}\right] \\
& \times K_{L}^{(V)}\left(x_{1}, x_{i} ; t_{1}-t_{i}\right) \tilde{V}\left(x_{1}\right) \ldots K_{L}^{(V)}\left(x_{n}, x_{n-1} ; t_{n}-t_{n-1}\right) \\
& \times \tilde{V}\left(x_{n}\right) K_{L}^{(V)}\left(x_{f}, x_{n} ; t_{f}-t_{n}\right)
\end{aligned}
$$

where $K_{L}^{(V)}$ is the fractional propagator for the unperturbed potential and again, it is assumed to be known. We have ordered time as $t_{i}<t_{1}<t_{2}<\ldots<t_{f}$ and paid attention to the fact that $K_{L}\left(t_{k}-t_{k-1}\right)$ is different from zero only if $t_{k}>t_{k-1}$.

Now for an arbitrary potential $V(x)$ with an additional $\delta$-perturbation, $W(x)=$ $V(x)-\gamma \delta(x-a)$, the path integral is given by

$$
\begin{aligned}
K_{L}^{(\delta)}\left(x_{f}, x_{i} ; t_{f}-t_{i}\right)= & K_{L}^{(V)}\left(x_{f}, x_{i} ; t_{f}-t_{i}\right)+\sum_{n=1}^{\infty}\left(\frac{-i \gamma}{\hbar}\right)^{n} \int_{t_{i}}^{t_{f}} d t_{n} \ldots \\
& \times \int_{t_{i}}^{t_{1}} d t_{1} K_{L}^{(V)}\left(a, x_{i} ; t_{1}-t_{i}\right) \ldots \\
& \times K_{L}^{(V)}\left(a, a ; t_{n}-t_{n-1}\right) K_{L}^{(V)}\left(x_{f}, a ; t_{f}-t_{n}\right) .
\end{aligned}
$$

The energy-dependent Green's function for the unperturbed system is given by

$$
G^{(V)}\left(x_{f}, x_{i} ; E\right)=\frac{i}{\hbar} \int_{0}^{\infty} d T e^{i E T / \hbar} K_{L}^{(V)}\left(x_{f}, x_{i} ; t_{f}-t_{i}\right), T=t_{f}-t_{i}
$$

We also introduce the Green's function, $G^{(\delta)}\left(x_{f}, x_{i} ; E\right)$ for the perturbed system in a similar manner as equation (8). The emerging geometric power series can be 
summed up due to the convolution theorem of the Fourier transformation, hence we have 6,7

$$
G^{(\delta)}\left(x_{f}, x_{i} ; E\right)=G^{(V)}\left(x_{f}, x_{i} ; E\right)-\frac{G^{(V)}\left(x_{f}, a ; E\right) G^{(V)}\left(a, x_{i} ; E\right)}{G^{(V)}(a, a ; E)-1 / \gamma},
$$

where it is assumed that $G^{(V)}(a, a ; E)$ actually exists. The energy levels $E_{n}$ of the perturbed system can be determined in a unique way by the denominator of $G^{(\delta)}\left(x_{f}, x_{i} ; E\right)$.

\section{The infinite square well with delta-perturbation}

We first consider a particle in a potential $V(x)$ defined as

$$
V(x)= \begin{cases}0, & |x| \leq l \\ \infty, & |x|>l\end{cases}
$$

The fractional quantum-mechanical propagator for this system was already obtained by Dong, 4

$$
K_{L}^{(V)}\left(x_{f}, x_{i} ; t_{f}-t_{i}\right)=\frac{1}{l} \sum_{n=1}^{\infty} \exp \left[-i E_{n}\left(t_{f}-t_{i}\right) / \hbar\right] \sin \left[k_{n}\left(x_{i}-l\right)\right] \sin \left[k_{n}\left(x_{f}-l\right)\right],
$$

where

$$
k_{n}=\frac{n \pi}{2 l}, \quad E_{n}=D_{\alpha} \hbar^{\alpha}\left|k_{n}\right|^{\alpha} .
$$

Solving the energy-dependent Green's function for this propagator yields

$$
G^{(V)}\left(x_{f}, x_{i} ; E\right)=\frac{1}{l} \sum_{n=1}^{\infty}\left(\frac{1}{E_{n}-E}\right) \sin \left[k_{n}\left(x_{i}-l\right)\right] \sin \left[k_{n}\left(x_{f}-l\right)\right] .
$$

Hence, from equation (9), the Green's function for the perturbed system is given by

$$
\begin{aligned}
G^{(\delta)}\left(x_{f}, x_{i} ; E\right)= & \frac{1}{l} \sum_{n=1}^{\infty}\left(\frac{1}{E_{n}-E}\right) \sin \left[k_{n}\left(x_{i}-l\right)\right] \sin \left[k_{n}\left(x_{f}-l\right)\right] \\
& -\frac{1}{l^{2}} \sum_{n=1}^{\infty} \sum_{m=1}^{\infty}\left(\frac{1}{E_{n}-E}\right)\left(\frac{1}{E_{m}-E}\right) \sin \left[k_{n}(a-l)\right] \sin \left[k_{n}\left(x_{f}-l\right)\right] \\
& \times \sin \left[k_{m}\left(x_{i}-l\right)\right] \sin \left[k_{m}(a-l)\right] \\
& \times\left[\frac{1}{l} \sum_{n=1}^{\infty}\left(\frac{1}{E_{n}-E}\right) \sin ^{2}\left[k_{n}(a-l)\right]-1 / \gamma\right]^{-1} .
\end{aligned}
$$

\section{Conclusion}

We have expanded the Lévy path integral for the fractional quantum propagator in a perturabation series. An analogous expansion with the Feynman path integral was obtained. From the expanded propagator, the energy dependent Green's function for the delta-perturbed infinite square well was also obtained. 


\section{References}

1. N. Laskin, "Fractional quantum mechanics," Phys. lett. A 268, pp. 268-305, 2000.

2. N. Laskin, "Fractional quantum mechanics and Lévy path integrals," Phys. Rev. E 62, pp. 3135-3145, 2000.

3. J. Dong and M. Xu, "Some solutions to the space fractional Schrödinger equation using momentum representation method," J. Math. Phys. 48, 072105 , 2007.

4. J. Dong, "Lévy path integral approach to the solution of the fractional Schrödinger equation with infinite square well," arXiv:1301.3009v1 [math-ph], 2013.

5. E. C. de Oliveira, F. S. Costa, and J. Vaz Jr., "The fractional Schrödinger equation for delta potentials," J. Math. Phys. 51, 123517, 2010.

6. C. Grosche, "Path integrals for potential problems wth $\delta$-function perturbation," J. Phys. A 23, 5205, 1990.

7. C. Grosche, "Path integraton via summation of perturbation expansions and applications to totally reflecting boundaries and potential steps," Phys. Rev. Lett. 71 (1), pp. $1-4,1993$. 